\title{
The temperature of an optically trapped, rotating microparticle
}

Paloma Rodríguez Sevilla, Yoshihiko Arita, Xiaogang Liu, Daniel Jaque, and Kishan Dholakia

ACS Photonics, Just Accepted Manuscript • DOI: 10.1021/acsphotonics.8b00822 • Publication Date (Web): 09 Aug 2018

Downloaded from http://pubs.acs.org on August 10, 2018

\section{Just Accepted}

"Just Accepted" manuscripts have been peer-reviewed and accepted for publication. They are posted online prior to technical editing, formatting for publication and author proofing. The American Chemical Society provides "Just Accepted" as a service to the research community to expedite the dissemination of scientific material as soon as possible after acceptance. "Just Accepted" manuscripts appear in full in PDF format accompanied by an HTML abstract. "Just Accepted" manuscripts have been fully peer reviewed, but should not be considered the official version of record. They are citable by the Digital Object Identifier (DOI®). "Just Accepted" is an optional service offered to authors. Therefore, the "Just Accepted" Web site may not include all articles that will be published in the journal. After a manuscript is technically edited and formatted, it will be removed from the "Just Accepted" Web site and published as an ASAP article. Note that technical editing may introduce minor changes to the manuscript text and/or graphics which could affect content, and all legal disclaimers and ethical guidelines that apply to the journal pertain. ACS cannot be held responsible for errors or consequences arising from the use of information contained in these "Just Accepted" manuscripts. 


\title{
The temperature of an optically trapped,
}

\section{rotating microparticle}

\author{
Paloma Rodríguez-Sevilla, ${ }^{* \dagger}$ Yoshihiko Arita, ${ }^{\dagger, \ddagger}$ Xiaogang Liu, "Daniel Jaque, ${ }^{\S}$ \\ and Kishan Dholakia*,†,\| \\ $\dagger$ †UPA, School of Physics and Astronomy, University of St Andrews, North Haugh, Fife, \\ KY16 9SS, United Kingdom. \\ $\ddagger$ Molecular Chirality Research Center, Chiba University, 1-33 Yayoi-cho, Inage-ku, \\ Chiba-shi 263-0022, Japan. \\ \Department of Chemistry National University of Singapore Science Drive 3, Singapore \\ 117543, Singapore. \\ $\S$ Fluorescence Imaging Group Departamento de Física de Materiales Universidad \\ Autónoma de Madrid 28049 Madrid, Spain and Nanobiology Group Instituto Ramón y \\ Cajal de Investigación Sanitaria Hospital Ramón y Cajal. Ctra. De Colmenar Viejo Km. \\ 9100, 28034 Madrid, Spain. \\ \|Graduate School of Science and Engineering, Chiba University, 1-33 Yayoi-cho, Inage-ku, \\ Chiba-shi 263-0022, Japan. \\ E-mail: prs8@st-andrews.ac.uk; kd1@st-andrews.ac.uk \\ Phone: +44 (0)1334 463184
}

\begin{abstract}
The measurement of temperature at the mesoscopic scale is challenging but important in a wide variety of research fields, including the investigation of single molecule and cell mechanics and interactions as well as fundamental studies in heat transfer and Brownian


dynamics on this scale. In this letter we present a route that determines temperature at the nano- to microscale with three independent measurements performed on a single trapped, rotating luminescent microparticle. We measure temperature changes using both the internal and external degrees of freedom, via (i) the upconverted luminescence, (ii) the rotation rate, and (iii) the Brownian dynamics of the particle. This novel tripartite approach allows us to cross-correlate the temperature for both the internal and external (center-of-mass) degree of freedom for the particle. In addition, our approach provides a measure of the temperature increase without the need of a precise knowledge of the particle dimensions, shape or any previous calibration of the sample or the experimental set-up. The developed technique opens up prospects for stringent tests of nanothermometry.

\section{Keywords}

optical trapping; upconverting particles; birefringence; optical torque; nanothermometry

The measurement of temperature at high resolution plays an important role in numerous processes, particularly at mesoscopic spatial scales. Micro and nanoparticle dynamics within a fluid are subject to Brownian motion, and as such, the particle's external degrees of freedom (translation and rotation) are strongly influenced by temperature due to their dependence on the viscosity of the surrounding medium.

Optical tweezers offer an ideal platform to study Brownian dynamics since the optical forces and torques acting on a trapped particle are counteracted and balanced by the forces and torques from the environment, which may also act as a method of heat dissipation. By recording the motion of a Brownian particle trapped in liquid, it is possible to probe the local fluid viscosity and its corresponding temperature around the particle. Recently much effort has been directed at cooling the center-of-mass (CoM) motion of trapped particles in vacuum, where the Brownian motion is controlled by optical fields, 14 with a view to 
reaching the quantum ground state. Within this context, the temperature of the particle is often assumed to be exclusively determined by its CoM motion (external degree of freedom) based on the equipartition theorem.

On the other hand, upconverting particles (UCPs), have recently brought a surge of interest in a variety of fields ranging from biological studies to quantum physics. UCPs are able to absorb two or more incident photons of relatively low energy and convert them into one emitted photon with higher energy, through a process known as upconversion (UC) $.5-7$ Importantly, the emission spectrum of a UCP encodes information of its internal temperature ${ }^{8}$ When a UCP is optically trapped with a continuous wave (cw) beam at an appropriate excitation wavelength it can be used as a remotely controlled thermometer $\stackrel{910}{9}$ Furthermore, depending on the doping type/content and the incident excitation wavelength, UCPs can be either refrigerated or heated, $\stackrel{11 \mid 12}{1}$ thus offer an interesting testbed to measure and control both the internal and CoM temperature.

Previous studies have shown that the internal temperature of a particle is in fact coupled to the CoM temperature. $\stackrel{413}{ }$ Recently, laser-induced refrigeration of an optically trapped Yb-doped UCPs has been reported both in liquid $\frac{14}{14}$ and vacuum. $\frac{11}{11}$ These studies show that the CoM temperature of the particle is comparable with its internal temperature within experimental errors. However, these studies do not offer any detailed analysis of how these internal and external temperatures compare in the context of micro- or nano-thermometry. Intriguingly, a recent study looked at coupling between internal and external degrees of freedom of a vacuum trapped nanoparticle. ${ }^{4}$ Heating by the laser and black-body radiation were attributed to lead to an internal temperature $(>1000 \mathrm{~K})$ well in excess of the CoM temperature $(<10 \mathrm{~K})$, even in the presence of parametric feedback cooling. Such studies are the cornerstone for future levitated hybrid optomechanics experiments. Importantly high internal particle temperatures can adversely affect studies of such particles at the classicalquantum interface and lead to a reduction in their performance as an ultra-precise sensor.

In this study we propose a novel tripartite method able to independently measure and 
correlate temperature changes from both the external and internal degrees of freedom for a single trapped UCP. Two different types of UCPs, one with high absorption and the other with lower absorption at the trapping wavelength, are employed. The dissipation of the absorbed energy occurs through non-radiative processes mediated by phonons in the material structure which produces a temperature increment only for the highly-absorbing particles. To demonstrate our approach we measure the temperature of a single UCP trapped in water. Firstly, the luminescent spectrum is recorded to determine the particle's internal temperature. This contrasts with other approaches 4 where a detailed knowledge of the particle material properties are required. This is then compared with the external (CoM) temperature of the UCP determined from the particle dynamics (translational and rotational degrees of freedom) directly linked to the viscosity changes of the environment.

A key attribute to distinguish our presented tripartite approach is that it does not require any knowledge of the particle physical properties, such as shape and size, or any calibration of the sample or the experimental system. Furthermore, our experimental errors are much smaller than those previously reported in aqueous media, 14,16 allowing us to provide a stringent approach for the analysis of heat transfer at the microscale for such optically trapped particles.

\section{Experimental}

Thermometers. Two differently doped UCPs were used in this study: $\mathrm{NaYbF}_{4}: \mathrm{Er}^{3+}, \mathrm{Nd}^{3+}$ and $\mathrm{NaYF}_{4}: \mathrm{Er}^{3+}, \mathrm{Yb}^{3+}$ microcrystals (see Methods for details). They present a similar disclike shape with hexagonal facets, as shown in the scanning electron microscope (SEM) images of Figures 1(a) and 1(b). In addition, both samples exhibit light emission in the visible range under near-infrared $788 \mathrm{~nm}$ excitation. Figures 1(c) and 1(d) show the normalized luminescence spectrum associated to the green emission of Er ions obtained from a single optically trapped $\mathrm{NaYbF}_{4}: \mathrm{Er}^{3+}, \mathrm{Nd}^{3+}$ and $\mathrm{NaYF}_{4}: \mathrm{Er}^{3+}, \mathrm{Yb}^{3+}$ particle, respectively. Importantly, the 
relative emission intensity of the bands centered around $520 \mathrm{~nm}$ and $540 \mathrm{~nm}$ is temperature dependent due to the change in the electronic population of the thermally-coupled excited

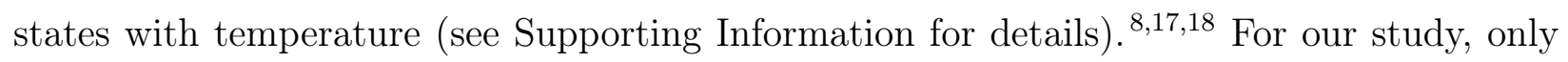
the highlighted bands in Figures 1(c) and 1(d) were used in order to reduce the experimental error in the determination of the thermal changes (see Supporting Information for details). In order to show the capability of the developed tripartite method to measure temperature changes for a trapped particle, we chose the two differently doped samples to present distinct light-to-heat conversion efficiencies (see Methods and Supporting Information for details). The excitation of $\mathrm{NaYbF}_{4}: \mathrm{Er}^{3+}, \mathrm{Nd}^{3+}$ microcrytals by $788 \mathrm{~nm}$ radiation produces an increase in they internal temperature. This is evidenced in Figure 1(c) where a clear change of the relative intensity of the highlighted bands takes place when laser power is increased. On the other hand, no temperature increase with laser power is seen for $\mathrm{NaYF}_{4}: \mathrm{Er}^{3+}, \mathrm{Yb}^{3+} \mathrm{UCPs}$ (see Figure 1(d)) which have lower absorption.

Both samples are positive uniaxial birefringent crystals, where the optical axis is perpendicular to the two hexagonal facets. .1920 Thus these particles can be rotated by a circularly polarized $(\mathrm{CP})$ beam. In addition, both types of particles are non-spherical. This affects the trapping properties of the particle. Due to the action of two different optical torques, the particle is stably trapped with its optical axis parallel to the polarization of the trapping laser, if linearly polarized (LP) light is used.20 Thus, the trapped particle is stably orientated with its longitudinal axis parallel to the propagation direction of the trapping beam. This means that, when $\mathrm{CP}$ light is used, the rotation axis is parallel to the longitudinal axis of the particle.

Thermometric techniques. The following described techniques (luminescence, rotation rate and trap stiffness methods) can provide a value for the thermal loading $\left(\mathrm{C}_{P}\right)$, which is the temperature increment per unit of power generated by the trapped particle. The temperature increase depends on the amount of light absorbed by the particle and the fraction of that light 
(a)

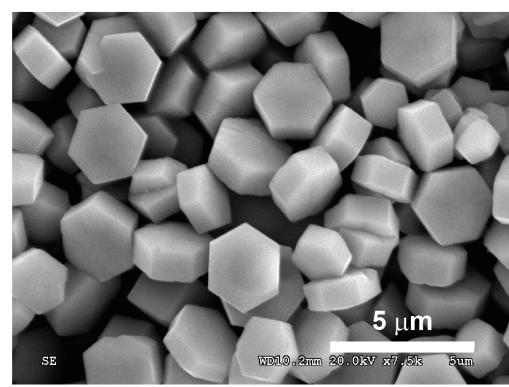

(c)

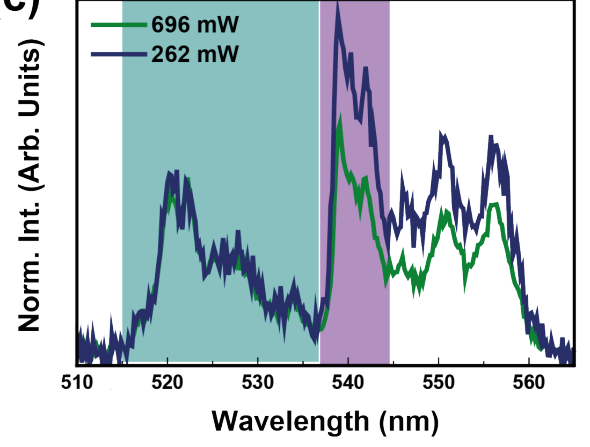

(b)

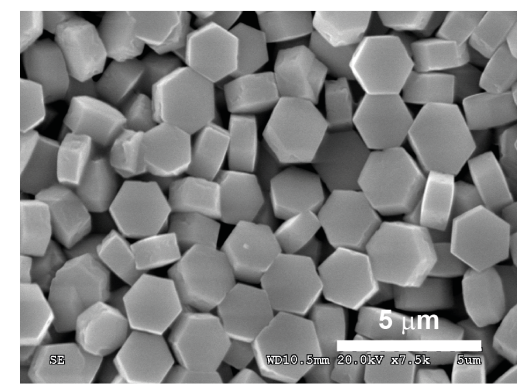

(d)

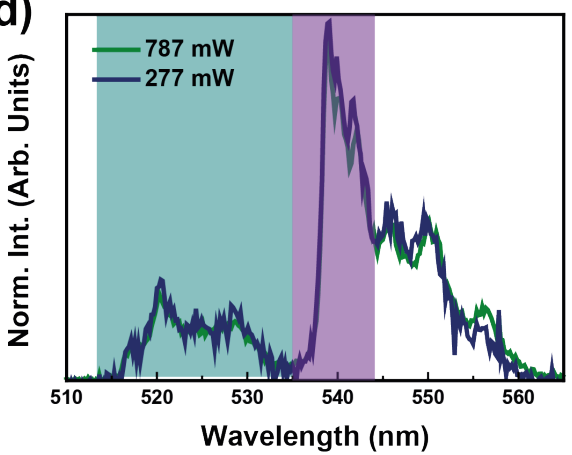

Figure 1: SEM images of (a) $\mathrm{NaYbF}_{4}: \mathrm{Er}^{3+}, \mathrm{Nd}^{3+}$ and (b) $\mathrm{NaYF}_{4}: \mathrm{Er}^{3+}, \mathrm{Yb}^{3+}$ particles and their corresponding luminescence spectra in (c) and (d), respectively, for two different excitation powers.

that is converted into heat through energy dissipation processes (see Supporting Information for details). Although both the absorption rate and energy dissipation are expected to depend on temperature, this dependence has been shown to be negligible in previous studies which show a linear temperature increment with laser power in the range of temperature explored here. ${ }^{12}$ Thus we assume a temperature increment $(T)$ with the laser power $(P)$ of the form: $T(P)=T_{0}+C_{P} P$, where $T_{0}$ is the initial temperature with no laser power applied (i.e. room temperature). We stress that the rotation rate and trap stiffness methods described below are independent of particle size and shape distinguishing this approach from previous, established routes for determination of CoM temperature.10141152121

Luminescence method. This method obtains a value of the thermal loading $\left(C_{P I}\right)$ from the changes in the intensity ratio $\left(\frac{I_{2}}{I_{1}}\right)$ of the two temperature-dependent emission bands: 


$$
\ln \left(\frac{I_{2}}{I_{1}}\right)=-C_{E} \frac{1}{T_{0}+C_{P I} P}+C_{I},
$$

where $C_{E}$ and $C_{I}$ are constants which include information of the thermalized energy levels involved in the radiative transitions (see Supporting Information for details). ${ }^{817}$ Thus, $C_{P I}$ can be determined from the evolution of $\ln \left(\frac{I_{2}}{I_{1}}\right)$ with $P$. It is worthy of note that, contrary to conventional thermometry studies, a prior calibration of the luminescence response with temperature is not needed since that information is included in the fitting parameters $C_{E}$ and $C_{I}$.

Rotation rate method. When a birefringent particle is trapped by a CP beam, an optical torque will act on it: $\tau_{\text {opt }}=\Delta \sigma P / \omega$, where $\Delta \sigma$ is the change in the degree of polarization and $\omega$ the angular frequency of light. The magnitude of $\tau_{\text {opt }}$ depends on the birefringence of the particle, which induces a phase retardation in the components of the CP light resulting in a change in the output polarization. In addition, optical torque transfer can be produced thorough absorption of the CP light by the particle. ${ }^{[22}$ Due to the Stokes drag torque counteracting $\tau_{\text {opt }}$, the trapped particle rotates at a terminal rotation rate: $\Omega=(\Delta \sigma P) /(\beta(\eta) \omega)$, where $\beta(\eta)$ denotes the rotational Stokes drag coefficient, which is dependent on the medium's vis$\operatorname{cosity}(\eta(T))$. Here we have used a tabulated dataset ${ }^{23}$ to obtain the temperature-dependent viscosity of water: $\eta(T)=A+B e^{-T / C}$, with $A=0.156 \pm 0.007 \mathrm{mPas}, B=1.37 \pm 0.02 \mathrm{mPas}$ and $C=41 \pm 1^{\circ} \mathrm{C}$. Therefore the dependence of $\Omega(P)$ can be written in the form:

$$
\Omega(P)=\frac{C_{R} P}{A+B e^{-\left(T_{0}+C_{P \Omega} P\right) / C}},
$$

where $C_{R}=\Delta \sigma /\left(\omega \beta^{\prime}\right)$, with $\beta^{\prime}=\beta / \eta$, is a constant that includes the characteristics of the particle and $\tau_{\text {opt }}$. The linear dependence of $T$ with the laser power has also been included. The thermal loading $\left(C_{P \Omega}\right)$ can be determined from the fitting of Eq. (2) to the experimental rotation rate as a function of the laser power. Therefore, here, $C_{P \Omega}$ can be directly obtained from $\Omega(P)$, while traditional studies require a prior knowledge of the drag coefficient of the 
particle (and therefore particle's size and shape) and the thermal profile in the surrounding medium in order to be able to fit the measured rotation rate to the theoretical model. 2124 In our case, particle's size and shape are included as the fitting parameter $C_{R}$.

Trap stiffness method. A particle trapped in an optical potential undergoes Brownian motion subject to the trap stiffness, which can be obtained by the analysis of its position power spectrum. $\cdot 25$ The corner frequency $\left(f_{c}\right)$ of the power spectrum determines the trap stiffness: $\kappa=2 \pi f_{c} \beta(T)$, which is also dependent on $T^{2126}$ and therefore provides a way to measure temperature. Correct determination of the trap stiffness requires knowledge of the thermal loading $\left(C_{P \kappa}\right)$. We determine its value by a numerical optimization routine which enforces the expected linear relationship between $\kappa$ and $P$ (see Supporting Information for details). This novel method presents several advantages in contrast to the traditional ways used to measure temperature from the particle's Brownian motion. Usually, power spectrum and equipartition method results are combined. ${ }^{15}$ However, former studies have shown that different trap stiffness calibration methods lead to a different value of the trap stiffness due to their particular limitations, $\stackrel{27}{27}$ and therefore, the combination of results from different methods introduces more uncertainty in the measurement of the temperature. Moreover, to obtain a value of $\kappa$ from the equipartition method, the QPD signal needs to be converted to displacement in distance units, rather than voltage, thus a calibration is needed. ${ }^{[13} \mathrm{In}$ addition, to obtain a value of $\kappa$ from the power spectrum, a knowledge of the drag coefficient of the particle (including size and shape) is required. In this case, we have used a theoretical translational drag coefficient for the disc-like particles: $\beta^{\prime}=\beta / \eta=6 \mathrm{Vf} / f_{0} R^{-2}$, where $V$ and $R$ are the volume and radius of the particle, respectively, and $f / f_{0}$ the Perrin friction factor. ${ }^{28}$ Nevertheless, this information is not necessary in our case since our procedure can be applied for a normalized value of $\kappa\left(\kappa / 2 \pi \beta^{\prime}=f_{c} \eta\right)$, since the drag coefficient is a constant that multiplies the viscosity of the medium. 


\section{Results and discussion}

Highly-absorbing particles. Figures 2(a-c) show the experimental data obtained from an optically trapped $\mathrm{NaYbF}_{4}: \mathrm{Er}^{3+}, \mathrm{Nd}^{3+}$ microparticle for the different techniques. The change in the intensity ratio with the laser power (Figure 2(a)) indicates an increment of the temperature of the particle according to a thermal loading of $C_{P I}=0.37 \pm 0.06{ }^{\circ} \mathrm{CmW}^{-1}$. Figure 2(b) shows the rotation rate as a function of the applied laser power. The best fit of the experimental data with Eq. (2) provides a thermal loading of $C_{P \Omega}=0.35 \pm 0.05^{\circ} \mathrm{CmW}^{-1}$. Finally, Figure 2(c) shows a superlinear behavior of $\kappa$, assuming a constant temperature $\left(T=22{ }^{\circ} \mathrm{C}\right.$ ) (green circles). The green line is a guide for the eye. Blue data correspond to the optimized linear relationship between $\kappa$ and $P$ for a thermal loading of $C_{P \kappa}=0.11 \pm$ $0.04{ }^{\circ} \mathrm{CmW}^{-1}$. All the results are summarized in Table 1 .

These thermal loadings of a single $\mathrm{NaYbF}_{4}: \mathrm{Er}^{3+}, \mathrm{Nd}^{3+}$ particle are one order of magnitude larger than those measured for colloidal solutions of Nd-doped nanoparticles, $\frac{12}{b u t}$ they are comparable to those reported for individual gold nanoparticles. $\frac{26 \mid 29}{2}$ This suggests high light-to-heat conversion efficiency of the studied particles (see Supporting Information for details). Due to this fact, temperatures above the boiling point of water were reached during experiments, as shown in Figure 3. We note that there are a number of reports showing lightexcited particles exceeding the boiling point of water at the standard pressure. ${ }^{29}$ However, it is often the case that no bubble formation was observed probably due to the increase of the critical temperature in the proximity of the particle (see Supporting Information for details). In addition, it is worth noting that no signs of particle disruption were observed even though its temperature can reach as high as $280^{\circ} \mathrm{C}$ during the trapping experiments (see Supporting Information for details). 

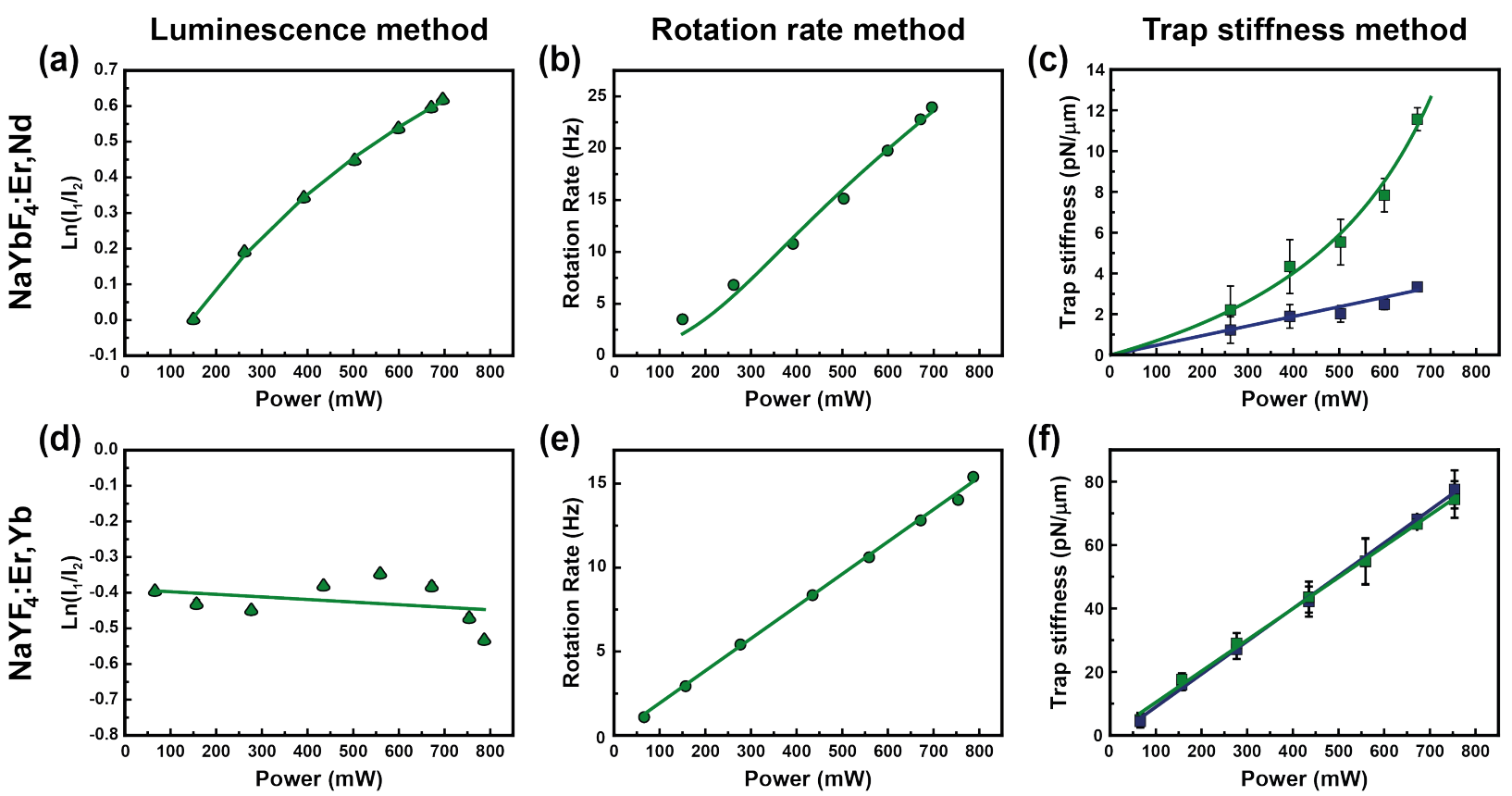

Figure 2: (a) Change in the intensity ratio as a function of the trapping power, (b) rotation rate as a function of the laser power and (c) trap stiffness as a function of the laser power when temperature is set to $22^{\circ} \mathrm{C}$ (green) and when temperature increment is considered (blue) for a $\mathrm{NaYbF}_{4}: \mathrm{Er}^{3+}, \mathrm{Nd}^{3+}$ microparticle. (d) Change in the intensity ratio as a function of the trapping power, (e) rotation rate as a function of the laser power and (f) trap stiffness as a function of the laser power when temperature is set to $22^{\circ} \mathrm{C}$ (green) and when temperature increment is consider (blue) for a $\mathrm{NaYF}_{4}: \mathrm{Er}^{3+}, \mathrm{Yb}^{3+}$ microparticle. Where not present, error bars are smaller than the symbols denoting the data points.

Table 1: Measured thermal loadings. ${ }^{a}$ This thermal loading was calculated from the luminescence thermal resolution $\left(0.5^{\circ} \mathrm{C}\right)$ divided by the maximum applied laser power $(\sim 800 \mathrm{~mW})$. All values are given in ${ }^{\circ} \mathrm{CmW}^{-1}$.

\begin{tabular}{ccc}
\hline Particle & $\mathrm{NaYbF}_{4}: \mathrm{Er}^{3+}, \mathrm{Nd}^{3+}$ & $\mathrm{NaYF}_{4}: \mathrm{Er}^{3+}, \mathrm{Yb}^{3+}$ \\
\hline Luminescence & $0.37 \pm 0.06$ & $<0.001^{a}$ \\
Rotation rate & $0.35 \pm 0.05$ & $0.001_{-0.001}^{+0.006}$ \\
Trap stiffness & $0.11 \pm 0.04$ & $0.007 \pm 0.003$ \\
\hline
\end{tabular}

Weakly-absorbing particles. The temperature increment produced by a optically trapped single $\mathrm{NaYF}_{4}: \mathrm{Er}^{3+}, \mathrm{Yb}^{3+}$ microparticle was also measured which is a particle with a lower absorption at $788 \mathrm{~nm}$ than $\mathrm{NaYbF}_{4}: \mathrm{Er}^{3+}, \mathrm{Nd}^{3+}$ particles. Results are shown in Figures $2(\mathrm{~d}-$ f) and Table 1. The thermal loading for the luminescence method could not be calculated because the temperature increment by the laser-induced heating is lower than the thermal resolution of the technique, as explained in the next section. The rotation rate analysis 


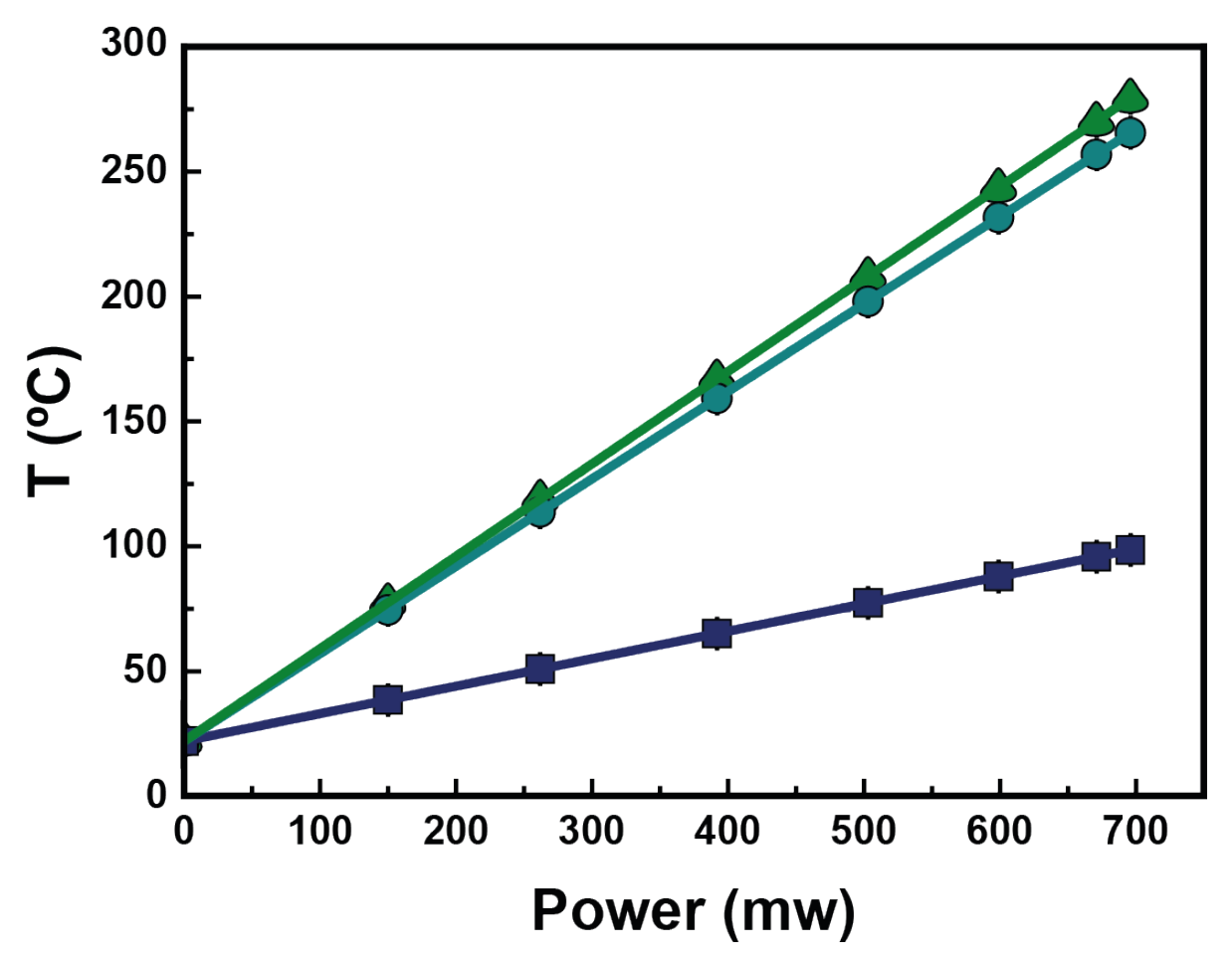

Figure 3: Temperature as a function of power measured for a $\mathrm{NaYbF}_{4}: \mathrm{Er}^{3+}, \mathrm{Nd}^{3+}$ microparticle by using the three thermometric methods: luminescence (green triangles), rotation rate (cyan circles), trap stiffness (navy squares). A room temperature of $22^{\circ} \mathrm{C}$ is assumed.

provides a thermal loading value that agrees with that estimated from luminescence thermometry. On the other hand, the trap stiffness measurements give a non-negligible thermal loading that deviates by an order of magnitude (see Table 1). This discrepancy could result from the fact that the trap stiffness method is very sensitive to the accuracy in the determination of $f_{c}$. Therefore, even in the absence of a temperature increment, the linearity of $\kappa$ can be affected by the uncertainty in the measurement of $f_{c}$, and the optimization process is able to find a value of $C_{P \kappa}$ which enhances the linear relationship between $\kappa$ and $P$.

We note that the results obtained for a non-heated $\mathrm{NaYF}_{4}: \mathrm{Er}^{3+}, \mathrm{Yb}^{3+}$ microparticle evidence that the main heating source in the experiments developed with a $\mathrm{NaYbF}_{4}: \mathrm{Er}^{3+}, \mathrm{Nd}^{3+}$ microcrystal is the particle itself. Water presents a low absorption coefficient ( $\alpha_{\text {abs }}=$ $\left.0.02 \mathrm{~cm}^{-132}\right)$ at $790 \mathrm{~nm}$, which is negligible in the present regime.

It is worth noticing that Figure 2 shows that $\mathrm{NaYF}_{4}: \mathrm{Er}^{3+}, \mathrm{Yb}^{3+}$ microparticles present larger trap stiffness and a lower rotation rate than $\mathrm{NaYbF}_{4}: \mathrm{Er}^{3+}, \mathrm{Nd}^{3+}$ microcrystals. We 
attribute this discrepancy to the different temperatures and absorption coefficients of the particles. In the case of the rotational motion, the temperature increment produces a reduction in the viscosity of the surrounding medium leading to a larger rotation rate for the $\mathrm{NaYbF}_{4}: \mathrm{Er}^{3+}, \mathrm{Nd}^{3+}$ microparticles. In addition, optical torque can be transferred through absorption producing an increase in the rotation rate for the highly-absorbing particles. The contrary effect is observed for the trap stiffness which is not only reduced as a result of the increment in the temperature, but also due to the larger absorption of the particles which increases the scattering force. Consequently, $\mathrm{NaYbF}_{4}: \mathrm{Er}^{3+}, \mathrm{Nd}^{3+}$ microparticles are trapped in a weaker optical potential region which leads to a reduction in the trap stiffness.

Thermal sensitivity and resolution. The thermal sensitivity $(S)$ is used to describe and compare the performance of different thermometric systems: $S=\frac{1}{C} \frac{d C}{d T}$, where $C$ is the parameter used to determine the changes in the temperature, i.e. intensity ratio $\left(\frac{I_{1}}{I_{2}}\right)$, trap stiffness $(\kappa)$ or rotation rate $(\Omega)$. Since both rotation rate and trap stiffness methods measure the changes in temperature through the viscosity of the surrounding medium, they present the same sensitivity $\left(2.0 \% \mathrm{~K}^{-1}\right)$, which is three times larger than that of the luminescence method $\left(0.66 \% \mathrm{~K}^{-1}\right)$. This latter case is within the range published for similar luminescence thermometers $\left(0.2-2.3 \% \mathrm{~K}^{-1}\right)$.

These thermal sensitivities can be used to obtain a value of the thermal resolution $(\delta T)$ of each technique: $\delta T=\frac{1}{S} \frac{\delta C}{C}$, where $\delta C$ is the uncertainty in the determination of the parameter $C$. The thermal resolutions are $0.5^{\circ} \mathrm{C}, 0.03^{\circ} \mathrm{C}$, and $0.1^{\circ} \mathrm{C}$ for the luminescence, rotation rate, and trap stiffness measurements, respectively. Here, the thermal resolution of the luminescence method was used to estimate the internal thermal loading (see Table 1 ) for the weakly-absorbing particle. The thermal resolution achieved by the combined methods ensures a higher accuracy in the determination of the thermal changes than previously published studies of optically heated or cooled particles in aqueous media. ${ }^{14}$ [16 This capability will improve future analysis of the CoM and internal temperatures and their coupling. 


\begin{abstract}
Different degrees of freedom. Furthermore, this tripartite approach allows us for the
\end{abstract} first time to simultaneously analyze the temperatures obtained from three different degrees of freedom of the particle: internal, rotational and translational. We note that it has been suggested, both experimentally and theoretically, by previous studies that the medium temperature probed by the dynamics of a particle can yield different temperature depending on whether the rotational or translational motion is considered. $\frac{15 \mid 21}{12}$ Moreover, the internal temperature of the particle is typically higher than the temperature of the surrounding medium.

In the case of highly-absorbing particles $\left(\mathrm{NaYbF}_{4}: \mathrm{Er}^{3+}, \mathrm{Nd}^{3+}\right)$, Table 1 shows that the thermal loading measured from the luminescence and the rotation methods are in good agreement within the experimental error, whereas the trap stiffness method exhibits a lower temperature (see also Figure 3). This discrepancy can be tentatively explained by taking into account that the temperature of the fluid varies with the distance from the particle surface, which means that there will be a non-uniform distribution of fluid viscosity around the particle. As mentioned above, previous studies suggest that the particle experiences a different temperature depending on whether we consider rotational or translational motion, which is known as "hot Brownian motion" (HBM). 15]33 state affects the Brownian dynamics of the particle depending upon whether the rotational or translational degree of freedom is under consideration. ${ }^{15 / 21 / 34 \mid 35}$ When rotating, the particle dynamics are affected by the liquid in close proximity to it which is at a temperature close to its internal, whereas when it translates the particle explores regions at a lower temperature. In other words, the fluid velocity field is more localized near the particle for rotation than for translation, therefore the effective temperature for rotational motion is higher than for translational motion. $\frac{35}{35}$ This thermal difference between the translational and rotational degrees of freedom would be enhanced for larger temperature increments and in media with a lower heat conductivity (such as low pressure gas or vacuum) where heat dissipation is reduced. $\frac{413}{43}$ Even though former studies have shown consistent results with those presented here, there 
is not a clear explanation of the discrepancy between the different degrees of freedom. The technique here presented can be used in future studies on the dynamics of optically trapped particles in HBM conditions to give a better insight of the problem in different media.

Finally, our results show that the temperature of the internal degree of freedom can only be assessed by using the luminescence of the particle. The rotational degree of freedom is affected by the superficial temperature of the particle which is, under our experimental uncertainty, equal to that of the internal degree of freedom. On the other hand, the translational degree of freedom exhibits a lower temperature than that of the particle itself due to the HBM. Our study shows that, in non-equilibrium thermal conditions, the Brownian dynamics, i.e. the external degrees of freedom, of the particle do not give a real value of the particle temperature, whereas only the luminescence, i.e. the internal degree of freedom, allows an access to the internal temperature of the particle.

\section{Conclusions}

In summary, the temperature of an optically trapped upconverting particle has been measured by studying its internal and external degrees of freedom. The internal degree of freedom has been experimentally assessed through the temperature-dependent luminescence of the particle, while the rotational and translational degrees of freedom were analyzed through the rotation rate and the trap stiffness of the particle, respectively. The higher thermal resolution in comparison with former studies has allowed a detailed study between these three independent methods. Both the internal and rotational degrees of freedom yielded the same effective temperature, while the translational motion exhibited a lower temperature in the non-thermal equilibrium state. These results are in good agreement with the hot Brownian motion to which the particle is subjected. Moreover, we note that any non-spherical particle will present non-zero off-diagonal terms in its hydrodynamic friction tensor that couples translational and rotational motion. $\frac{36}{}$ This is a minor effect not considered in the 


\section{Methods and materials}

Particle preparation. The hydrothermal procedure used to synthesize the two different types UCPs is described elsewhere. $\frac{910}{10}$ The first sample is composed of colloidal $\mathrm{NaYbF}_{4}$ microcrystals, doped with a $2 \%$ of trivalent erbium and a $10 \%$ of trivalent neodymium ions, while the other is a colloidal solution of $\mathrm{NaYF}_{4}: 0.5 \% \mathrm{Er}^{3+}, 5 \% \mathrm{Yb}^{3+}$ microcrystals.

Optical trapping set-up. We use a standard optical tweezers set-up with a Ti:Sapphire laser (Coherent, Mira 900-F) tuned to a wavelength of $788 \mathrm{~nm}$. This wavelength is chosen because it is able to both excite the UC luminescence and induce absorption within the trapped particle, while minimizing any absorption by water. The LP beam was focused by a high numerical aperture (NA) microscope objective lens (Nikon, E Plan, 100× NA=1.25, in oil) to trap individual particles suspended in deionized water. A quarter-wave plate was placed immediately before the microscope objective in order to switch the beam's polarization 
from LP to CP or vice versa. For the analysis of the particle dynamics, the forward scattered light by the trapped particle was collected using a condenser lens (Mituyoto, M Plan Apo, $20 \times \mathrm{NA}=0.42)$ and detected by a quadrant photodiode (QPD, First Sensor, QP50-6SD2,-3 dB at $150 \mathrm{kHz}$ ). A CCD camera (Basler, piA640-210gm) was used for the visualization of the trapped particle. Finally, a compact, fiber-coupled spectrometer (Oceanoptics, USB4000) was used for the analysis of the particle's luminescence. The very same condenser lens was used to collect the luminescence and a shortpass filter (Thorlabs, FESH0700) was placed at the entrance of the fiber to block the laser light from reaching the detector.

Experimental protocol. The following procedure was applied for the determination of the thermal loading through the developed tripartite thermometric technique. First, the trapped particle was set into rotation by a CP beam at different excitation powers up to $800 \mathrm{~mW}$. At each power, a rotation signal was recorded by the QPD for $6 \mathrm{~min}$. During that time, six luminescence spectra were measured every 1 min. Once the rotation experiment was completed, the same particle was trapped by an LP beam to measure the trap stiffness for the same power range using the QPD. At each power level, ten consecutive measurements were performed in order to obtain reliable statistics, together with six consecutive measurements of luminescence spectra following the trap stiffness measurement.

\section{Author information}

P.R.S. and Y.A. performed the experiments and data analysis. All authors contributed to the development and planning of the project, interpretation and discussion of the data. P.R.S., Y.A. and K.D. wrote the manuscript. K.D. supervised the project. The authors declare no competing financial interests. 


\section{Acknowledgement}

P.R.S thanks the Spanish Ministerio de Economía y Competitividad (MINECO) for the "Promoción del talento y su Empleabilidad en $\mathrm{I}+\mathrm{D}+\mathrm{i}$ " state program for funding. We thank the UK Engineering and Physical Sciences Research Council for funding through grant EP/P030017/1 and MINECO for funding through grant MAT2016-75362-C3-1-R. COST Action CM1403 and UAM-Santander Yerun Projects are also acknowledged. This work was also Supported by Comunidad Autónoma de Madrid (B2017/BMD-3867RENIM-CM), by the European Commission (NanoTBTech). Work co-financed by European Structural and Investment Fund. Graham D. Bruce is acknowledge for technical support.

\section{Supporting Information Available}

The following file is available free of charge.

- Supp.pdf: Supporting information

This material is available free of charge via the Internet at http://pubs.acs.org/.

\section{References}

(1) Li, T.; Kheifets, S.; Raizen, M. G. Millikelvin cooling of an optically trapped microsphere in vacuum. Nature Physics 2011, \%, 527.

(2) Li, Y. L.; Millen, J.; Barker, P. F. Cooling the centre-of-mass motion of a silica microsphere. Proc. SPIE, Optical Trapping and Optical Micromanipulation XI 2014, 9164, 916404 .

(3) Bullier, N. P.; Pontin, A.; Barker, P. F. Millikelvin cooling of the center-of-mass motion of a levitated nanoparticle. Proc. SPIE, Optical Trapping and Optical Micromanipulation XIV 2017, 10347, 103471K. 
(4) Hebestreit, E.; Reimann, R.; Frimmer, M.; Novotny, L. Measuring the internal temperature of a levitated nanoparticle in high vacuum. Physical Review A 2018, 97, 043803.

(5) Dong, H.; Sun, L.-D.; Yan, C.-H. Basic understanding of the lanthanide related upconversion emissions. Nanoscale 2013, 5, 5703-5714.

(6) Zhou, B.; Shi, B.; Jin, D.; Liu, X. Controlling upconversion nanocrystals for emerging applications. Nat Nano 2015, 10, 924-936.

(7) Nadort, A.; Zhao, J.; Goldys, E. M. Lanthanide upconversion luminescence at the nanoscale: fundamentals and optical properties. Nanoscale 2016, 8, 13099-13130.

(8) Jaque, D.; Vetrone, F. Luminescence nanothermometry. Nanoscale 2012, 4, 4301-4326.

(9) Rodríguez-Sevilla, P.; Zhang, Y.; Haro-González, P.; Sanz-Rodríguez, F.; Jaque, F.; Solé, J. G.; Liu, X.; Jaque, D. Thermal Scanning at the Cellular Level by an Optically Trapped Upconverting Fluorescent Particle. Advanced Materials 2016, 28, 2421-2426.

(10) Rodríguez-Sevilla, P.; Lee, T.; Liang, L.; Haro-González, P.; Lifante, G.; Liu, X.; Jaque, D. Light-Activated Upconverting Spinners. Advanced Optical Materials 2018, 1800161.

(11) Rahman, A. T. M. A.; Barker, P. F. Laser refrigeration, alignment and rotation of levitated $\mathrm{Yb}^{3+}$ :YLF nanocrystals. Nature Photonics 2017, 11, 634-638.

(12) Ximendes, E. C.; Rocha, U.; Jacinto, C.; Kumar, K. U.; Bravo, D.; Lopez, F. J.; Rodriguez, E. M.; Garcia-Sole, J.; Jaque, D. Self-monitored photothermal nanoparticles based on core-shell engineering. Nanoscale 2016, 8, 3057-3066.

(13) Millen, J.; Deesuwan, T.; Barker, P.; Anders, J. Nanoscale temperature measurements using non-equilibrium Brownian dynamics of a levitated nanosphere. Nature Nanotechnology 2014, 9, 425. 
(14) Roder, P. B.; Smith, B. E.; Zhou, X.; Crane, M. J.; Pauzauskie, P. J. Laser refrigeration of hydrothermal nanocrystals in physiological media. Proceedings of the National Academy of Sciences 2015, 112, 15024-15029.

(15) Hajizadeh, F.; Shao, L.; Andrén, D.; Johansson, P.; Rubinsztein-Dunlop, H.; Käll, M. Brownian fluctuations of an optically rotated nanorod. Optica 2017, 4, 746-751.

(16) Zhou, X.; Smith, B. E.; Roder, P. B.; Pauzauskie, P. J. Laser Refrigeration of Ytterbium-Doped Sodium-Yttrium-Fluoride Nanowires. Advanced Materials 2016, 28, $8658-8662$.

(17) Zhou, S. S.; Deng, K. M.; Wei, X. T.; Jiang, G. C.; Duan, C. K.; Chen, Y. H.; Yin, M. Upconversion luminescence of $\mathrm{NaYF}_{4}: \mathrm{Yb}^{3+}, \mathrm{Er}^{3+}$ for temperature sensing. Opt. Commun. 2013, 291, 138-142.

(18) Baral, S.; Johnson, S. C.; Alaulamie, A. A.; Richardson, H. H. Nanothermometry using optically trapped erbium oxide nanoparticle. Appl. Phys. A 2016, 122, 1-8.

(19) Chen, P.; Song, M.; Wu, E.; Wu, B.; Zhou, J.; Zeng, H.; Liu, X.; Qiu, J. Polarization modulated upconversion luminescence: single particle vs. few-particle aggregates. Nanoscale 2015, 7, 6462-6466.

(20) Rodríguez-Sevilla, P.; Zhang, Y.; de Sousa, N.; Marqués, M. I.; Sanz-Rodríguez, F.; Jaque, D.; Liu, X.; Haro-González, P. Optical Torques on Upconverting Particles for Intracellular Microrheometry. Nano Letters 2016, 16, 8005-8014.

(21) Arita, Y.; Richards, J. M.; Mazilu, M.; Spalding, G. C.; Skelton Spesyvtseva, S. E.; Craig, D.; Dholakia, K. Rotational Dynamics and Heating of Trapped Nanovaterite Particles. ACS Nano 2016, 10, 11505-11510.

(22) Friese, M. E. J.; Enger, J.; Rubinsztein-Dunlop, H.; Heckenberg, N. R. Optical angular- 
momentum transfer to trapped absorbing particles. Physical Review A 1996, 54, 15931596.

(23) Kestin, J.; Sokolov, M.; Wakeham, W. A. Viscosity of liquid water in the range $-8^{\circ} \mathrm{C}$ to $150^{\circ}$ C. J. Phys. Chem. Ref. Data 1978, 7, 941-948.

(24) Parkin, S. J.; KnÂûner, G.; Nieminen, T. A.; Heckenberg, N. R.; RubinszteinDunlop, H. Picoliter viscometry using optically rotated particles. Physical Review E 2007, 76, 041507.

(25) Berg-Sørensen, K.; Flyvbjerg, H. Power spectrum analysis for optical tweezers. Review of Scientific Instruments 2004, 75, 594-612.

(26) Seol, Y.; Carpenter, A. E.; Perkins, T. T. Gold nanoparticles: enhanced optical trapping and sensitivity coupled with significant heating. Opt. Lett. 2006, 31, 2429-2431.

(27) Sarshar, M.; Wong, W. T.; Anvari, B. Comparative study of methods to calibrate the stiffness of a single-beam gradient-force optical tweezers over various laser trapping powers. Journal of Biomedical Optics 2014, 19.

(28) Erb, R. M.; Segmehl, J.; Charilaou, M.; Loffler, J. F.; Studart, A. R. Non-linear alignment dynamics in suspensions of platelets under rotating magnetic fields. Soft Matter 2012, 8, 7604-7609.

(29) Kyrsting, A.; Bendix, P. M.; Stamou, D. G.; Oddershede, L. B. Heat Profiling of ThreeDimensionally Optically Trapped Gold Nanoparticles using Vesicle Cargo Release. Nano Lett. 2011, 11, 888-892.

(30) Lehmuskero, A.; Ogier, R.; Gschneidtner, T.; Johansson, P.; Käll, M. Ultrafast Spinning of Gold Nanoparticles in Water Using Circularly Polarized Light. Nano Letters 2013, 13, 3129-3134. 
(31) Baffou, G.; Polleux, J.; Rigneault, H.; Monneret, S. Super-Heating and Micro-Bubble Generation around Plasmonic Nanoparticles under cw Illumination. The Journal of Physical Chemistry C 2014, 118, 4890-4898.

(32) Kedenburg, S.; Vieweg, M.; Gissibl, T.; Giessen, H. Linear refractive index and absorption measurements of nonlinear optical liquids in the visible and near-infrared spectral region. Opt. Mater. Express 2012, 2, 1588-1611.

(33) Rings, D.; Schachoff, R.; Selmke, M.; Cichos, F.; Kroy, K. Hot Brownian Motion. Physical Review Letters 2010, 105, 090604.

(34) Ruijgrok, P. V.; Verhart, N. R.; Zijlstra, P.; Tchebotareva, A. L.; Orrit, M. Brownian Fluctuations and Heating of an Optically Aligned Gold Nanorod. Physical Review Letters 2011, 107, 037401.

(35) Rings, D.; Chakraborty, D.; Kroy, K. Rotational hot Brownian motion. New Journal of Physics 2012, 14, 053012.

(36) Kraft, D. J.; Wittkowski, R.; ten Hagen, B.; Edmond, K. V.; Pine, D. J.; LÃúwen, H. Brownian motion and the hydrodynamic friction tensor for colloidal particles of complex shape. Physical Review E 2013, 88, 050301. 
Figure 1: SEM images of (a) $\mathrm{NaYbF}_{4}: \mathrm{Er}^{3+}, \mathrm{Nd}^{3+}$ and (b) $\mathrm{NaYF}_{4}: \mathrm{Er}^{3+}, \mathrm{Yb}^{3+}$ particles and their corresponding luminescence spectra in (c) and (d), respectively, for two different excitation powers.

Figure 2: (a) Change in the intensity ratio as a function of the trapping power, (b) rotation rate as a function of the laser power and (c) trap stiffness as a function of the laser power when temperature is set to $22^{\circ} \mathrm{C}$ (green) and when temperature increment is consider (blue) for a $\mathrm{NaYbF}_{4}: \mathrm{Er}^{3+}, \mathrm{Nd}^{3+}$ microparticle. (d) Change in the intensity ratio as a function of the trapping power, (e) rotation rate as a function of the laser power and (f) trap stiffness as a function of the laser power when temperature is set to $22^{\circ} \mathrm{C}$ (green) and when temperature increment is consider (blue) for a $\mathrm{NaYF}_{4}: \mathrm{Er}^{3+}, \mathrm{Yb}^{3+}$ microparticle. Where not present, error bars are smaller than the symbols denoting the data points.

Figure 3: Temperature as a function of power measured for a $\mathrm{NaYbF}_{4}: \mathrm{Er}^{3+}, \mathrm{Nd}^{3+}$ microparticle by using the three thermometric methods: luminescence (green triangles), rotation rate (cyan circles), trap stiffness (navy squares). A room temperature of $22^{\circ} \mathrm{C}$ is assumed. 


\section{Graphical TOC Entry}

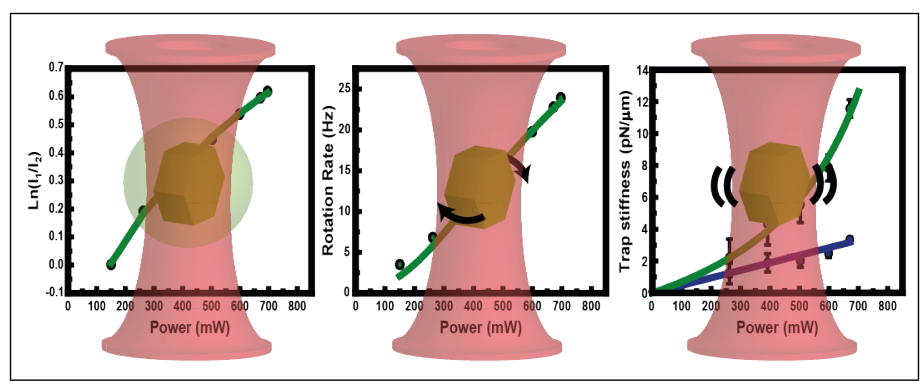

